\title{
Material Properties for Quantitative Thermoelastic Stress Analysis of Composite Structures
}

\author{
R.K. Frühmann ${ }^{\text {a }}$, S. Sambasivam ${ }^{b}$, J.M. Dulieu-Barton ${ }^{c}$ and S. Quinn ${ }^{d}$ \\ School of Engineering Sciences, University of Southampton, Southampton SO17 1BJ, UK \\ arkf@soton.ac.uk, 'sshamala@soton.ac.uk, cjanice@soton.ac.uk, ${ }^{\mathrm{d}}$ s.quinn@soton.ac.uk
}

Keywords: Thermoelastic stress analysis (TSA), composite materials, strain witness

\begin{abstract}
The sensitivity of the thermoelastic response to variations in the fibre volume fraction, resin material and manufacturing route is assessed. To quantify any effects a comprehensive materials testing programme has been conducted to obtain coefficients of thermal expansion, specific heat, density and the elastic properties, which is described in detail in the paper. The work is focused on attempting to ascertain if the source of the response is from the isotropic resin rich layer or from the orthotropic substrate. It is also demonstrated that small variations in material properties can have a significant effect on the calculated thermoelastic response.
\end{abstract}

\section{Introduction}

Thermoelastic stress analysis (TSA) [1] is a well established experimental technique based on the measurement of small changes in temperature on the surface of a cyclically loaded component. It is usual to obtain the temperature change using a sensitive infra-red focal plane array detector system, thereby providing non-contact, full-field stress data.

The theoretical equation that allows TSA to be applied to orthotropic composite materials was derived by Stanley and Chan [2] as:

$$
\Delta T=\frac{-T}{\rho C_{p}}\left(\alpha_{1} \Delta \sigma_{1}+\alpha_{2} \Delta \sigma_{2}\right)
$$

where $\Delta T$ is the small thermoelastic temperature change, $\Delta \sigma_{1}$ and $\Delta \sigma_{2}$ are the stresses in the surface ply principal material directions, $T$ is the absolute surface temperature, $\rho$ is the density, $C_{p}$ is the specific heat at constant pressure and $\alpha$ is the coefficient of thermal expansion (CTE) in the principal material directions.

In this work it is convenient to use the reformulation of Eq. 1 in terms of strain [1]:

$$
\Delta T=\frac{-T}{\rho C_{p}}\left([\alpha]_{1,2}^{T}[Q]_{1,2}[J][\Delta \varepsilon]_{L, T}\right)
$$

where $Q$ is the stiffness, $J$ is a geometric transformation relating the principal material directions (1, 2) and global component $(L, T)$ orientations and $\Delta \varepsilon$ is the change in strain in the laminate principal material directions.

The manufacturing processes used to produce composite components typically result in a resin rich layer on the surface of a component. Since the resins used in composite materials are poor conductors, even a thin surface resin layer can act as a 'strain witness' [3, 4]. In these cases the thermoelastic response from the resin layer can be related to the strains in the laminate by [3, 4]: 


$$
\Delta T=\frac{-T}{\rho C_{p}}\left(\frac{\alpha E}{1-v} \Delta\left(\varepsilon_{1}+\varepsilon_{2}\right)\right)
$$

where $E$ is the Young's modulus, $v$ is the Poisson's ratio, $\varepsilon$ is the strain (in this case the subscripts 1 and 2 indicate principal strains) and the material properties are that of the resin.

In the present paper three types of glass/epoxy composite material are investigated. The difference in the material is primarily the manufacturing technique and the resin, as the glass fibre used in all of the materials is E-glass, which has very repeatable material properties. The three materials are liquid resin infused (RI) glass mat, autoclave consolidated (AC) glass/epoxy prepreg and oven consolidated (OC) glass/epoxy prepreg. The results presented for the OC material is from earlier work by the authors [4]. Three different epoxy resins are used in the materials, i.e. Prime 20 LV supplied by Gurit for the RI, UD001/00 from Primco for the AC and SE84 from Gurit for the OC. To isolate the influence of the resin, nominally identical glass/epoxy unidirectional (UD) materials have been made using the three manufacturing processes. The manufacturing processes produce materials with different fibre volume fractions, which result in different mechanical and thermal properties. However, if the thermoelastic response is that of the epoxy resin surface layer as given by equation (3), then for the same applied strain sum and surface temperature, $\Delta T$ should be the same for each specimen. The purpose of this paper is to investigate if this is the case and also how the resin type and processing can affect the thermoelastic response. To investigate the response of the resin alone, specimens made from Prime $20 \mathrm{LV}$ are used as a benchmark for comparison. A further point is that the resin layer thickness for the three materials is different; this is also considered in the analysis. The experimental work in the paper demonstrates the importance of accurate material property values when using TSA to assess the performance of composite materials and the requirement for a calibration procedure to obviate extensive mechanical testing to obtain material properties.

\section{Test specimen manufacture and material properties}

The test specimens used in this work were of a UD tensile type with the fibres orientated at $0^{\circ}$. For the RI, the specimen material was produced using the weft fibres from $2 \times 2$ twill E-glass fabric and infusing the Prime $20 \mathrm{LV}$ epoxy resin in conjunction with a fast hardener. This material was manufactured from 10 plies, resulting in a laminate $1.6 \mathrm{~mm}$ thick. The process produced an irregular surface resin layer varying in thickness from 10 to $90 \mu \mathrm{m}$. The AC material was manufactured from 6 plies of material in an autoclave for one hour at a temperature of $125^{\circ} \mathrm{C}$ and a pressure of 3 bar and then post cured in an oven at a temperature of $150{ }^{\circ} \mathrm{C}$ for a period of 16 hours. Two panels of this material were made, one for specimens with a fibre orientation of $0^{\circ}$ and one for specimens with a fibre orientation of $90^{\circ}$, which were $1.6 \mathrm{~mm}$ and $2.1 \mathrm{~mm}$ thick respectively. The surface resin thickness variation of this material was less than the RI material (20 to $40 \mu \mathrm{m})$. The composite material used for the OC test specimens is described in detail in [4] and comprised 13 layers of a unidirectional E-glass/epoxy (SE84) pre-impregnated material. The material was consolidated under vacuum pressure for one hour and then cured for four hours at a temperature of $80^{\circ} \mathrm{C}$, resulting in a laminate that was $3.5 \mathrm{~mm}$ thick with an average resin surface layer thickness of $29 \mu \mathrm{m}$.

The resin only test specimens were produced by mixing the Prime 20 LV resin and the hardener and then pouring into a mould to produce individual specimens, $40 \mathrm{~mm}$ wide by $10 \mathrm{~mm}$ thick. Great care was taken to ensure that air bubbles did not form in the mixing and pouring processes, so that voids in the specimens were minimised. The material was left to cure at room temperature and pressure in the mould for 12 hours and then post cured at a temperature of $50^{\circ} \mathrm{C}$ for 16 hours.

In evaluating the thermoelastic response of a material the properties of interest are given in Eqs. 2 and 3 and are namely: $\rho, C_{p}$, the CTEs in the principal material directions, and the material stiffness, $Q$, made up of the longitudinal and transverse Young's moduli and the major and minor Poisson's ratios. A summary of the material property data for each specimen type is provided in 
Table 1 along with the volume fraction, $V_{f}$, and the thickness of the resin rich surface layer. Table 1 shows rows labelled 'measured', 'calculated', 'manufacturer' and 'literature'. Measured refers to properties that have been obtained by measurement. Calculated refers to properties that have been obtained by calculation using the volume fraction and the properties of the constituent materials as given in any standard reference on composites, e.g. [5]. Manufacturer refers to properties provided in the manufacturers' data sheets or through private discussion. Literature refers to values obtained from published work.

\section{Table 1: Material property summary}

\begin{tabular}{|c|c|c|c|c|c|c|c|c|c|c|c|}
\hline \multirow{2}{*}{\multicolumn{2}{|c|}{$\begin{array}{l}\text { Material manufacturing } \\
\text { type }\end{array}$}} & \multirow{2}{*}{$\begin{array}{r}V_{f} \\
(\%) \\
\end{array}$} & \multirow{2}{*}{$\frac{\rho}{\left(\mathrm{kg} \mathrm{m}^{-3}\right)}$} & \multirow{2}{*}{$\frac{C_{p}}{\left(\mathrm{~J} \mathrm{~kg}^{-1} \mathrm{~K}^{-1}\right)}$} & $E_{1}$ & $E_{2}$ & $v_{12}$ & $v_{21}$ & $\alpha_{1}$ & $\alpha_{2}$ & \multirow{2}{*}{$\frac{\text { Resin layer }}{(\mu \mathrm{m})}$} \\
\hline & & & & & \multicolumn{2}{|c|}{$(\mathrm{GPa})$} & & & \multicolumn{2}{|c|}{$\left(10^{-6} \mathrm{~K}^{-1}\right)$} & \\
\hline \multirow{2}{*}{ RI } & Measured & 58.4 & & 828 & 48.6 & 15.2 & 0.288 & 0.107 & 8.5 & 24.8 & $39 \pm 24$ \\
\hline & Calculated & & 1982 & 983 & 43.9 & 8.9 & 0.285 & 0.058 & 7.0 & 35.1 & \\
\hline \multirow{3}{*}{$\mathrm{AC}$} & Measured & 52.7 & 1880 & 843 & 34.2 & 10.0 & 0.325 & 0.100 & 9.0 & 31.0 & $30 \pm 9$ \\
\hline & Manufacturer & $\begin{array}{c}62 \\
{[6]}\end{array}$ & & & $\begin{array}{l}45 \\
{[6]}\end{array}$ & & & & & & \\
\hline & Calculated & & 1900 & 1006 & 40.0 & 8.0 & 0.297 & 0.059 & 7.5 & 39.2 & \\
\hline \multirow{3}{*}{$\begin{array}{l}\mathrm{OC} \\
{[4]}\end{array}$} & Measured & 66.4 & & & 36.8 & 8.4 & 0.250 & 0.050 & & & 29 [7] \\
\hline & Literature & & 1846 [7] & 882 [8] & & & & & $\begin{array}{l}6.0 \\
{[9]}\end{array}$ & $\begin{array}{c}35.0 \\
{[9]}\end{array}$ & \\
\hline & Calculated & & 2100 & 887 & 49.3 & 10.0 & 0.267 & 0.054 & 6.5 & 29.4 & \\
\hline \multirow{3}{*}{ Resin } & Measured & - & 1156 & 1225 & 4.0 & - & 0.405 & - & 57.5 & - & \\
\hline & Manufacturer & - & $\begin{array}{l}1153 \\
{[10]}\end{array}$ & 970 [11] & $\begin{array}{c}3.0 \\
{[10]}\end{array}$ & - & $\begin{array}{c}0.440 \\
{[12]} \\
\end{array}$ & - & $\begin{array}{l}65.0 \\
{[12]}\end{array}$ & - & \\
\hline & Literature & - & $\begin{array}{l}1170 \\
{[13]}\end{array}$ & $1040[8]$ & $\begin{array}{c}3.7 \\
{[14]}\end{array}$ & - & $\begin{array}{c}0.400 \\
{[13]} \\
\end{array}$ & - & & - & \\
\hline $\begin{array}{l}\text { E- } \\
\text { glass }\end{array}$ & Literature [15] & - & 2570 & 810 & 72.4 & - & 0.200 & - & 5.0 & - & \\
\hline
\end{tabular}

$V_{f}$ was obtained using micrographs of the cross sections of the material. It was estimated that the fibre volume fractions were $58.4 \%, 52.7 \%$ and $66.4 \%$ for the RI, $0^{\circ} \mathrm{AC}$ and $\mathrm{OC}$ material respectively. It should be noted here that for the $\mathrm{AC}$ material the volume fraction was much less than that suggested by the manufacturers for the recommended cure cycle.

$\rho$ was measured for the AC material and the resin by measuring the mass and the volume of a 40 x $40 \mathrm{~mm}$ specimen, $1.6 \mathrm{~mm}$ thick for the composite material and $7 \mathrm{~mm}$ thick for the resin. Two specimens of each were used and the average used to define $\rho$. The calculated values for the RI and AC material were obtained using the fibre volume fraction and the densities of the constituent material, resin and glass fibre. The calculated $\rho$ of the AC material is just over $1 \%$ greater than the measured value. The values for the resin alone correspond to within $1.5 \%$, with the measured value in between the manufacturer and literature value. $C_{p}$ was measured using differential scanning calorimetry (DSC) using specimens approximately $2 \times 2 \times 1.6 \mathrm{~mm}$ thick for the RI material and $4 \mathrm{x}$ $4 \times 1.5 \mathrm{~mm}$ thick for the AC material. For the RI material the calculated and measured values differ by $19 \%$. For the AC material the measured and calculated values differ again by $19 \%$. The measured values for the RI and AC laminates are within $2 \%$ of each other. The literature and calculated values for the OC material are very close. The measured value of $C_{p}$ for the resin is greater than the manufacturers $(-21 \%)$ and the literature values $(-15 \%)$.

The test specimens used to obtain the elastic properties were cut from the manufactured panel as strips of material approximately $26 \mathrm{~mm}$ wide for the RI specimens, $21 \mathrm{~mm}$ for the $0^{\circ} \mathrm{AC}$ specimens, $41 \mathrm{~mm}$ for the $90^{\circ} \mathrm{AC}$ specimens and $40 \mathrm{~mm}$ for OC specimens. In all cases end tabs were used; in the case of the RI and AC specimens these were not scarfed and were bonded after the specimens had been cut. For the RI material the end tabs were made from sheet steel whilst for the AC material cross-ply composite end tabs were used. The OC specimens were manufactured according to ASTM D3039-76 and scarfed composite end tabs were bonded to the panel before cutting into specimens. Young's modulus and Poisson's ratio values were obtained from standard quasi-static tensile tests 
using an Instron servo-mechanical test machine. For the RI and AC specimens strain data was obtained from strain gauge rosettes and the global stress in the laminate was calculated using data from the test machine load cell and the measured cross-sectional area. For the OC material long gauge extensometers were used and the strain derived as given in [4]. The measured values for $E_{1}$ vary by $30 \%$, with the $\mathrm{AC}$ being the lowest. The calculated $E_{1}$ value for the RI is lower than the measured, but is greater for both the $\mathrm{AC}$ and $\mathrm{OC}$. The high value for the RI material could be attributed to a slow infusion and cure as the laboratory temperature was low (approximately $15{ }^{\circ} \mathrm{C}$ ) during the panel manufacture. The very low value for the AC could be attributed to the low $V_{f}$, but the calculated value using this $V_{f}$ is at the lower end of the manufacturers range. There is a $25 \%$ variation in $E$ of the resin with the measured value being the greatest. The measured $E_{2}$ values differ by $45 \%$ with the RI giving the greatest value, which is much higher than the calculated value; the calculated and measured values for the other two processes are within $20 \%$. The high value for $E_{2}$ is once again attributable to the processing conditions. The measured values of $v_{12}$ vary by $23 \%$ compared to $53 \%$ for $v_{21}$. In the RI material there is very close agreement between the measured and calculated values of $v_{12}$; this is not the case for the other two processing methods. The values of $v_{21}$ differ by approximately $50 \%$ for the RI and AC but are very close for the OC process. $v$ for the resin differs by $10 \%$ between the measured, manufacturers and literature values.

The CTEs were obtained by heating smaller specimens $(40 \mathrm{~mm}$ square $\mathrm{x}$ the relevant panel thickness) from 20 to $40{ }^{\circ} \mathrm{C}$ and comparing the strain gauge output with that from specialist strain gauges supplied by Vishay Measurements Group UK Ltd (CEA-06-125UT-350) mounted on a copper reference material of known CTE [16]. The measured $\alpha_{1}$ values for the RI and AC laminates are within $6 \%$ of each other but differ by a greater amount with the calculated values. The literature and calculated values for the OC laminate are very close. The measured value of $\alpha$ for the resin is $12 \%$ less than the values quoted by the manufacturers and in the literature. $\alpha_{2}$ values for the RI and AC laminates differ by $20 \%$ and vary considerably from the calculated values. Likewise for the OC the difference between the calculated and quoted values is large.

The work in this section of the paper clearly demonstrates the large variation in material properties for composite materials, even those that are nominally the same. The work illustrates that simply calculating a value based on the constituent materials properties will cause large errors and that values quoted in the literature and by manufacturers can differ significantly from the measured properties. Therefore, in the remainder of the paper the measured properties will be used, wherever possible, to calculate the thermoelastic response from each material using Eq. 2 or Eq. 3.

\section{Thermoelastic work}

A Silver 480M system, manufactured by Cedip Infrared Systems, was used to collect the TSA data from the RI and AC material. This is a solid state cooled system with a 320 by 256 pixel detector array; the system was fitted with a $27 \mathrm{~mm}$ infra-red lens. The specimens that were used to obtain the elastic material properties were also used for the TSA tests. The specimens had an orthogonal strain gauge rosette mounted on them, so that the strain sum was monitored during the TSA tests. An Instron servo-hydraulic test machine provided the cyclical loading at a frequency of $10 \mathrm{~Hz}$ (AC and $\mathrm{OC}$ ) and $30 \mathrm{~Hz}(\mathrm{RI})$. The required load to achieve an applied strain sum of approximately $1600 \mu \varepsilon$ was calculated prior to testing and verified by the strain gauge readings (see Tables 2 and 3). This applied strain gave a reasonable thermoelastic temperature change $(\Delta T)$ without the chance of introducing any fatigue damage during the tests [4]. The TSA readings were taken directly from the specimens, as the surface resin layer provided high and uniform surface emissivity. The measured $\Delta T$ values are given in Tables 2 and 3 in the column marked 'Mea.' and were obtained by taking an average from an array of data from the central portion of each specimen. The maximum variation in the data was around $16 \%$ for the $\mathrm{RI}$ material and $13 \%$ for the $\mathrm{AC}$ material, perhaps indicating the variation in resin thickness. The OC values were taken directly from [4], where values were only provided for the longitudinal direction. The Cedip system is such that it 
provides the magnitude of the temperature change and hence in the tables $\Delta T$ is shown as a positive value.

Table 2: Predicted and measured thermoelastic response of the UD material in the longitudinal direction

\begin{tabular}{|c|c|c|c|c|c|c|c|c|c|c|c|}
\hline & $\Delta \varepsilon_{L}$ & $\Delta \varepsilon_{T}$ & $\Delta \varepsilon_{L}+\Delta \varepsilon_{T}$ & \multirow{2}{*}{$\begin{array}{c}T \\
(\mathrm{~K})\end{array}$} & \multicolumn{3}{|c|}{$\Delta T(\mathrm{~K})$} & \multicolumn{2}{|c|}{$\Delta T / \Delta \varepsilon_{L} T\left(\mu \varepsilon^{-1}\right)$} & \multicolumn{2}{|c|}{$\Delta T /\left(\Delta \varepsilon_{L}+\Delta \varepsilon_{T}\right) T\left(\mu \varepsilon^{-1}\right)$} \\
\hline & \multicolumn{3}{|c|}{$(\mu \varepsilon)$} & & Comp. & Resin & Mea. & Comp. & Mea. & Resin & Mea. \\
\hline RI & 2312 & -665 & 1647 & 296 & 0.17 & 0.13 & 0.12 & 0.25 & 0.19 & 0.27 & 0.25 \\
\hline $\mathrm{AC}$ & 2264 & -581 & 1683 & 295 & 0.14 & 0.13 & 0.15 & 0.21 & 0.22 & 0.27 & 0.30 \\
\hline OC [4] & 2190 & -527 & 1663 & 291 & 0.09 & 0.13 & 0.14 & 0.14 & 0.22 & 0.27 & 0.29 \\
\hline
\end{tabular}

Table 3: Predicted and measured thermoelastic response of the UD material in the transverse direction

\begin{tabular}{|c|c|c|c|c|c|c|c|c|c|c|c|}
\hline & $\Delta \varepsilon_{L}$ & $\Delta \varepsilon_{T}$ & $\Delta \varepsilon_{L}+\Delta \varepsilon_{T}$ & \multirow{2}{*}{$\begin{array}{c}T \\
(\mathrm{~K}) \\
\end{array}$} & \multicolumn{3}{|c|}{$\Delta T(\mathrm{~K})$} & \multicolumn{2}{|c|}{$\Delta T / \Delta \varepsilon_{L} T\left(\mu \varepsilon^{-1}\right)$} & \multicolumn{2}{|c|}{$\Delta T /\left(\Delta \varepsilon_{L}+\Delta \varepsilon_{T}\right) T\left(\mu \varepsilon^{-1}\right)$} \\
\hline & \multicolumn{3}{|c|}{$(\mu \varepsilon)$} & & Comp. & Resin & Mea. & Comp. & Mea. & Resin & Mea. \\
\hline RI & 1777 & -170 & 1607 & 296 & 0.12 & 0.13 & 0.12 & 0.23 & 0.24 & 0.27 & 0.26 \\
\hline $\mathrm{AC}$ & 1738 & -114 & 1624 & 292 & 0.10 & 0.13 & 0.10 & 0.20 & 0.21 & 0.27 & 0.22 \\
\hline $\mathrm{OC}[4]$ & 1684 & -84 & 1600 & 291 & 0.09 & 0.13 & - & 0.18 & - & 0.27 & - \\
\hline
\end{tabular}

In Tables 2 and 3 calculated values of $\Delta T$ are provided based on the material properties given in Table 1. The column marked 'Comp.' uses Eq. 2 and the orthotropic composite material properties as follows: calculated $\rho$, measured $C_{p}$, measured $\alpha_{1}$ and $\alpha_{2}$, measured values for the elastic material properties $\left(E_{1}, E_{2}, v_{12}, v_{21}\right)$ for the RI and likewise for the $\mathrm{AC}$, except that $\rho$ was measured. In the case of the OC material [4] $\rho, C_{p}, \alpha_{1}$ and $\alpha_{2}$ were obtained from the literature and $E_{1}, E_{2}, v_{12}, v_{21}$ were measured. The column marked 'Resin' in Tables 2 and 3 provides calculated $\Delta T$ values for the resin rich layer using Eq. 3. In all three cases, the material properties were the measured values for the resin given in Table 1.

The $\Delta T$ values given in Tables 2 and 3 are dependent on the applied strain and the specimen surface temperature. To make these comparable it was decided to normalise for strain and temperature. For an orthotropic analysis this is not straight forward as in a tensile specimen this will be depend on $v_{L T}$ [4]. For the longitudinal specimens $v_{L T}$ is equivalent to $v_{12}$ and for the transverse specimen is equivalent to $v_{21}$. In Table 1 it can be seen that $v_{12}$ varies from 0.25 to 0.33 but in the case of $v_{21}$ the RI and AC values are very close together. As the Poisson's ratios are close for each material the quantity $\Delta T / \Delta \varepsilon_{L} T$ provides a good means of comparison. Assuming isotropic properties the normalisation is straightforward and the last two columns in Tables 2 and 3 provide the quantity $\Delta T /\left(\Delta \varepsilon_{L}+\Delta \varepsilon_{T}\right) T$ as the means of comparison.

It is clear from the results in Tables 2 and 3 that the thermoelastic response from materials made from different manufacturing procedures is different, but the differences are not significant. Therefore the data provides an opportunity to ascertain if the source of the thermoelastic response in composite components is from the resin rich layer or the orthotropic substrate. As accurate material properties are available for the resin of the orthotropic materials it should be a simple matter to make a comparison of the values in Tables 2 and 3 and draw a conclusion. From Table 2 it seems clear that the resin is the source of the response with the calculated and experimental values of $\Delta T /\left(\Delta \varepsilon_{L}+\Delta \varepsilon_{T}\right) T$ being within $9 \%$ of each other compared to $25-35 \%$ difference in the $\Delta T / \Delta \varepsilon_{L} T$ for the RI and $\mathrm{OC}$ values. In fact the average measured value, of $0.28 \mu \varepsilon^{-1}$, corresponds almost exactly to the calculated value. However, the measured and calculated values of $\Delta T / \Delta \varepsilon_{L} T$ for the $\mathrm{AC}$ material are very close to each other. In Table 3 the transverse data for the RI material indicates that the source of the response could be from either the resin or the substrate. This is not surprising as in this orientation the material is dominated by the resin. However, the results for the AC material show that it is more likely the response is from the orthotropic substrate. It is unfortunately the case 
for glass/epoxy that the two fibre orientations used here give very similar theoretical responses with $\Delta T / \Delta \varepsilon_{L} T$ equal to $0.25 \mu \varepsilon^{-1}$ and $0.23 \mu \varepsilon^{-1}$ for the longitudinal and transverse orientations respectively for the RI material, $0.21 \mu \varepsilon^{-1}$ versus $0.20 \mu \varepsilon^{-1}$ for the AC material and $0.14 \mu \varepsilon^{-1}$ versus $0.18 \mu \varepsilon^{-1}$ for the OC material. It can be concluded that glass/epoxy is not the most suitable material for evaluating this effect. A further and more interesting conclusion is that the thermoelastic response of glass/epoxy composites approximate to that of an isotropic material, making analysis much more straightforward. To confirm this, current work is examining different lay-ups of glass/epoxy material with off axes plies.

\section{Closure}

This paper has shown the importance of obtaining reliable measured material properties when using TSA to assess the stress in composite materials. Although not explored in this paper the need for a calibration routine based on the measured thermoelastic response is essential to avoid extensive mechanical testing. Most importantly the paper has demonstrated that the response from a glass/epoxy composite material is most likely to be from the resin rich surface layer. However the tests were inconclusive in the sense that the orthotropic material has a very similar response in the $0^{\circ}$ and $90^{\circ}$ material directions.

\section{References}

[1] J.M. Dulieu-Barton and P. Stanley: Journal of Strain Analysis Vol. 33 (1998), p. 93.

[2] P. Stanley and W.K. Chan: Journal of Strain Analysis Vol. 23 (1988), p. 137.

[3] G. Pitarresi, M.S. Found and E.A. Patterson: Composites Science and Technology Vol. 65 (2005), p. 269.

[4] T.R. Emery, J.M. Dulieu-Barton, J.S. Earl and Cunningham, P.R.: Composites Science and Technology, Vol. 68 (2008), p. 743.

[5] I.M. Daniel and O. Ishai: Engineering Mechanics of Composites Materials (Oxford University Press, USA 1994).

[6] Technical Data Sheet, UD001/00, Primco, 2005.

[7] P.R. Cunningham, J.M. Dulieu-Barton, A.G. Dutton and R.A. Shenoi: Key Engineering Materials Vol. 52 (2002), p. 483.

[8] G. Kalogiannakis, D.V. Hemelrijck and G.V. Assche: J of Composite Materials Vol. 38 (2004), p. 163.

[9] N. Harwood and W.M. Cummings (Eds.): Thermoelastic Stress Analysis (IOP Publishing Ltd., England 1991).

[10] Technical Data Sheet, Prime 20LV Epoxy Infusion System, Gurit, 2006.

[11]Personal communication, R. Crutchlow (Gurit) to R.K. Frühmann (University of Southampton), 2007.

[12]Personal communication, R. Crutchlow (Gurit) to R.K. Frühmann (University of Southampton), 2006.

[13]J.M. Dulieu-Smith and P. Stanley: J of Material Processing Technology Vol. 78 (1998), p. 75.

[14] Technical Data Sheet, 135 Resin/229 Hardener, Pro-Set Inc., 2007.

[15] Information on http://www.matweb.com

[16] Technical note TN-513-1F, Vishay Micro Measurements, 2007. 